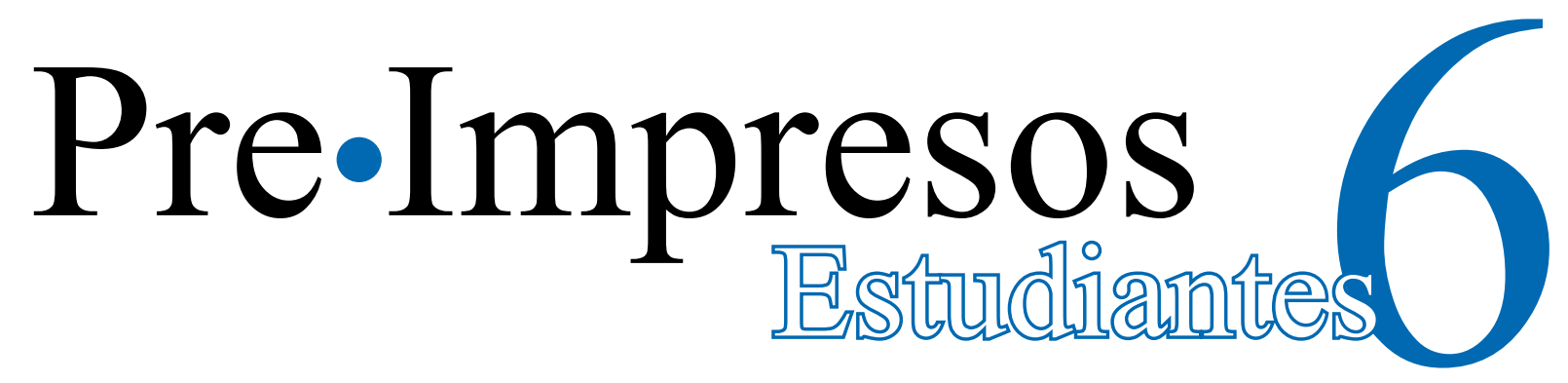

Facultad de Ciencia y Tecnología Departamento de Tecnología 2011

\title{
Scilab como herramienta de aplicación en sistemas de comunicación
}

Manuel Francisco Romero

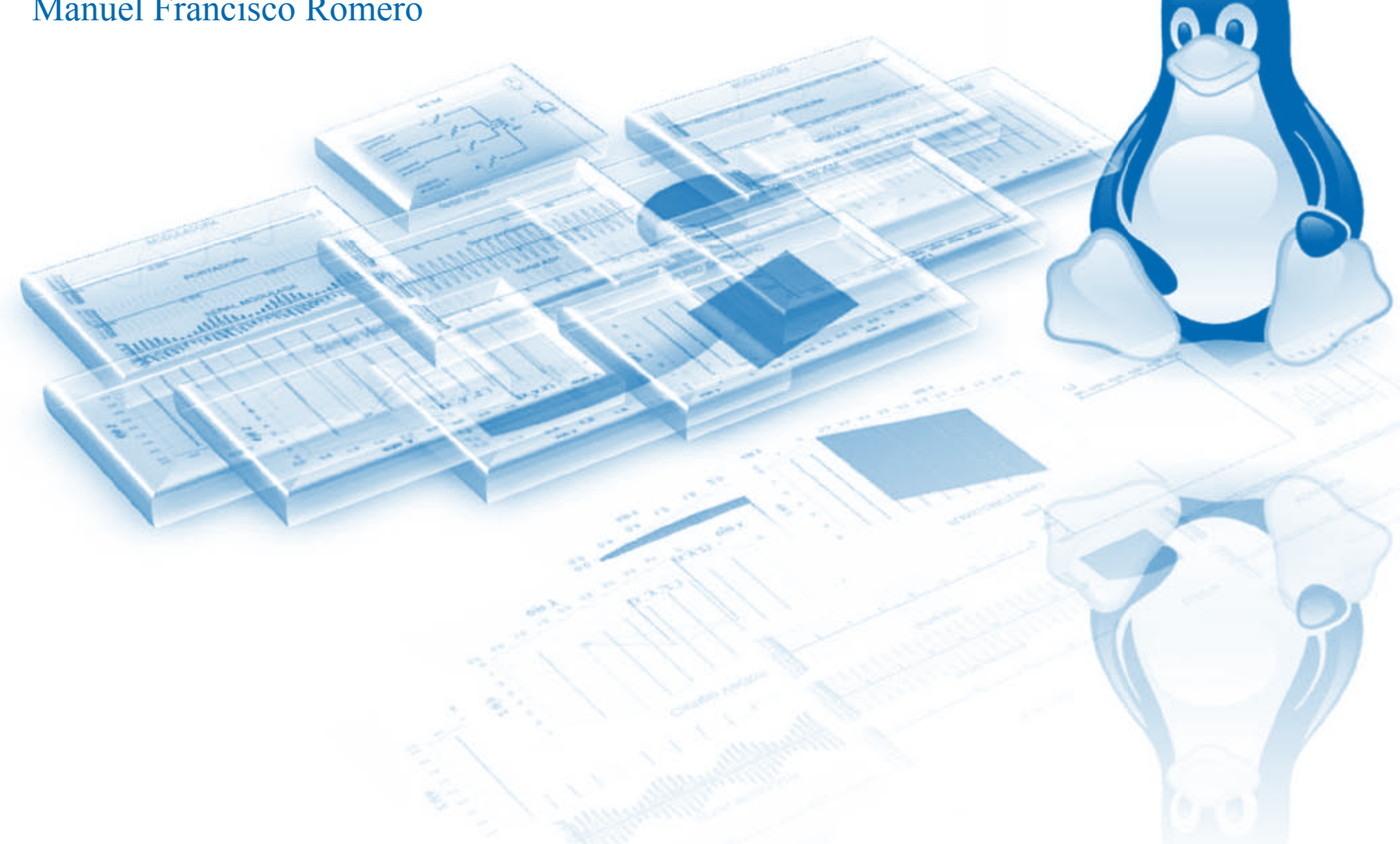




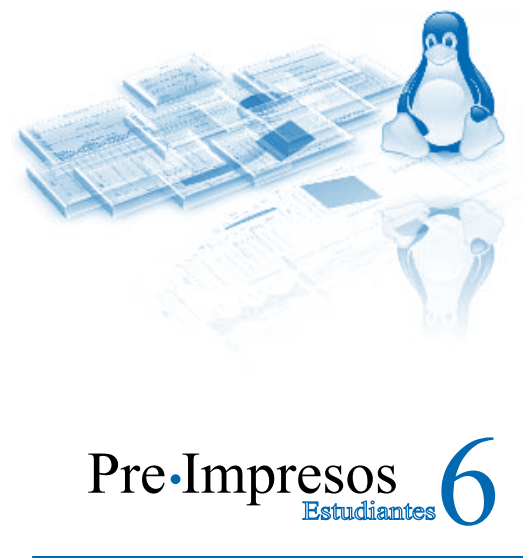

Juan Carlos Orozco Cruz

Rector

Edgard Alberto Mendoza Prada

Vicerrector Académico

María Ruth Hernández Martínez

Vicerrectora Administrativa y Financiera

José Domingo Garzón Garzón

Vicerrector de Gestión Universitaria

Facultad de Ciencia y Tecnología

Departamento de Tecnología

Nelson Otálora López

Jefe de Departamento

Diego Acero Soto

Coordinador Licenciatura en Electrónica

Jimmy Ramírez Cano

Supervisión de Contenido

Juan Carlos Bustos Gómez

Coordinador Editorial

(C) Universidad Pedagógica Nacional

(C) Manuel Francisco Romero

Imagen de portada

Scilab como herramienta de aplicación en sistemas de comunicación

Artículos publicados en diferentes medios escritos y referenciados en cada uno de los textos.

Diseño y Preparación editorial Universidad Pedagógica Nacional Grupo de Comunicaciones Corporativas 2011

Impreso en

Bogotá, Colombia
Scilab como herramienta de aplicación en sistemas de comunicación

$\begin{array}{lr}\text { Scilab como herramienta de aplicación } & \\ \text { en sistemas de comunicación } & \mathbf{1} \\ \text { Introducción } & \mathbf{2} \\ \text { Propuesta } & \mathbf{3} \\ \quad \text { Tipos de software } & 3 \\ \quad \text { Scilab } & \mathbf{4} \\ \text { Desarrollo } & \mathbf{5} \\ \text { Manual } & \mathbf{7} \\ \text { Sistemas de comunicaciones II } & 10 \\ \text { Conclusiones } & 12\end{array}$

\section{Presentación}

De acuerdo con la Misión de la Universidad Pedagógica Nacional, el objetivo de esta publicación es resaltar la importancia de la socialización de las ideas en el campo de las ciencias y su enseñanza; como contribución al fortalecimiento de la docencia y la investigación en educación, tendiendo puentes entre los saberes especializados y la cultura en general. Esto resulta pertinente y significativo en la formación de nuevas generaciones de maestros de ciencias e investigadores en pedagogía, desde campos disciplinares específicos, quienes en su futura práctica profesional afrontarán retos y circunstancias diversos, que el entorno social del país le plantea a la educación.

La serie Pre-Impresos es una iniciativa editorial dirigida a la comunidad académica en general, que divulga la producción intelectual de los estudiantes de la Facultad de Ciencia y Tecnología de la UPN, destacando las experiencias y reflexiones respecto de los temas propios del quehacer disciplinar y pedagógico de los autores. La participación está abierta a todos los integrantes de la comunidad estudiantil que deseen publicar sus trabajos a través de este medio; no hay restricción alguna en cuanto al formato de escritura, número de páginas o tema, con la salvedad de aquellos que estén fuera de los intereses propios de la actividad de la Facultad.

\section{Información:}

pre_impresos@pedagogica.edu.co

jcbustos@pedagogica.edu.co

Departamento de Física - UPN

Teléfonos: (57) (1) 3471190 / 5941894 Ext. 242 


\section{Pre.Impresos 6}

\section{Scilab como herramienta de aplicación en sistemas de comunicación}

\author{
Manuel Francisco Romero \\ Universidad Pedagógica Nacional \\ Licenciado en Electrónica \\ manuelfox189@gmail.com
}

\section{Abstract}

The main aspects to elaborate a manual which implement virtual experimental practices through the use of the Scilab, a specialized free software, are presented in this article. This proposal, besides to offer an alternative to carry out actual practices, has an educational value since it involves new elements to interpret the experiences. When integrating the concept of "dominions of representation" in the accomplishment of the laboratories, the manual elaborated exalts various aspects of the process graphical, instrumental, linguistic, analytical and from tools, facilitating the learning processes and, in addition, avoiding the difficulties to perform and multiply the experience, relating to the cost and possibilities of access.

Key words

Scilab, virtual labs, free and proprietary software, domains of representation, communication systems.

\section{Resumen}

Este artículo presenta los principales aspectos que se tuvieron en cuenta para la elaboración de un manual para la implementación de prácticas experimentales virtuales mediante el uso de un software libre especializado, como Scilab. Esta propuesta, además de ofrecer una alternativa a la realización de prácticas presenciales, tiene un valor pedagógico, ya que involucra nuevos elementos para la interpretación de las experiencias. Al integrar el concepto de "dominios de representación" en la realización de los laboratorios, el manual pone de relieve diferentes aspectos de los procesos gráficos, instrumentales, lingüísticos, analíticos y de herramientas, facilitando los aprendizajes $y$, además, soslayando las dificultades para realizar y replicar la experiencia, relativas al costo y las posibilidades de acceso.

\begin{tabular}{l|l} 
Palabras claves & $\begin{array}{l}\text { Scilab, laboratorios virtuales, soft- } \\
\text { ware libre y propietario, dominios } \\
\text { de representación, sistemas de } \\
\text { comunicaciones }\end{array}$
\end{tabular} 


\section{Introducción}

El presente artículo muestra el desarrollo de un manual para las asignaturas de Comunicaciones I y II de la Licenciatura en Electrónica de la Universidad Pedagógica Nacional. Surge como una alternativa para subsanar las dificultades habituales en la realización de prácticas experimentales, tales como el número insuficiente de equipos para atender la alta demanda y los altos costos de los implementos de laboratorio, con la dificultad adicional para repetir la práctica en el laboratorio de la institución.

Los ambientes virtuales de aprendizaje constituyen una herramienta que facilita la elaboración de material digital para la realización de laboratorios virtuales, posibilitando a estudiantes y profesores desarrollar las mismas competencias y actividades que en un laboratorio real, al mismo tiempo que dinamiza los procesos de enseñanza y se adecúa a las necesidades actuales de los centros educativos.

En este sentido, el manual ha permitido elaborar actividades para ser realizadas dentro y fuera del aula, afianzando aspectos teórico prácticos de las asignaturas, pues los componentes gráficos y de simulación invitan al estudiante a explorar y modificar los elementos de las actividades propuestas, lo cual les permite, incluso, observar el comportamiento de fenómenos físicos con mucho más detalle que en un laboratorio real, además hace posible compartir experiencias con el grupo de estudiantes y de docentes. De esta forma, se contribuye a mejorar la comprensión, a fortalecer el trabajo colaborativo y a cualificar los procesos de aprendizaje. Igualmente, facilita la labor del docente en el diseño de prácticas de laboratorio.

La implementación de los "dominios de representación" ${ }^{11}$ es una estrategia de enseñanza aprendizaje que consiste en desagregar un fenómeno en diferentes etapas o fases para su estudio $y$, de esta forma, ampliar la comprensión sobre el mismo, como principal propósito. Entre los dominios de representación considerados se cuentan los siguientes:

\section{Dominio gráfico}

Consiste en la representación de un fenómeno en un entorno gráfico con el fin de fortalecer conceptos de simulación. Apoyado en la visualización del fenómeno, representado gráficamente, el estudian- te debe poner en juego sus conocimientos sobre los conceptos básicos de las temáticas del curso para la interpretación, modificación y construcción de criterios suficientes, que son referentes a los elementos manipulados, como la frecuencia, la amplitud, fase y modulación, entre otros.

\section{Dominios analíticos}

Consiste en la interpretación de un problema partiendo de un enunciado, un gráfico, una expresión matemática o la combinación de algunas de estas representaciones para concebir posibles soluciones al problema propuesto. En el proceso de búsqueda de un método adecuado para su solución, el estudiante mejora la comprensión del problema, a la vez que desarrolla sus habilidades analíticas.

\section{Dominio simbólico}

Se refiere al estudio de fenómenos físicos, que se representa mediante modelos matemáticos, actividad que exige al estudiante claridad en los conceptos y manejo de las expresiones matemáticas.

\section{Dominio instrumental}

Representa el manejo de los conceptos matemáticos, habilidades de uso y selección de instrumentos y procesos aritméticos que se describen por medio de un algoritmo, dando soluciones a problemas numéricos que requieren del uso de herramientas tecnológicas como la calculadora, el software especializado, entre otros, que permitan simular y graficar situaciones experimentales.

\section{Dominio lingüístico}

Se refiere a los elementos lingüísticos propios del área temática específica, conocimiento que implica la profundización del tema para mejorar la compresión, la cual va aparejada con la apropiación de las terminologías utilizadas en cada proceso. Es decir, involucran la precisión léxica asociada a la comprensión profunda de un tema de estudio específico.

Cabe señalar que los diferentes dominios de representación descritos se pueden combinar, no es necesario utilizarlos de forma lineal, pueden encontrarse de forma implícita en cada actividad que se realice en una práctica de laboratorio.

\footnotetext{
1. Propuesta desarrollada por el profesor Guillermo Gómez de la Universidad Pedagógica Nacional, para la realización de las prácticas de laboratorio en la asignatura Circuitos I de la Licenciatura en Electrónica.
} 


\section{Pre.Impresos $\underset{\text { Estudiantes }}{6}$}

\section{Propuesta}

El uso de herramientas informáticas como el software libre y propietario ha permitido elaborar, a través de instrucciones y algoritmos, elementos de simulaciones y gráficos que contribuyen a mejorar procesos de enseñanza al facilitar la manipulación y observación de los "objetos" de estudio de una manera que difícilmente podríamos experimentar en una práctica pedagógica tradicional. Estas herramientas apoyan la labor docente, en especial en aquellos espacios académicos que requieren la realización de prácticas de laboratorio, pues favorece en los aprendices la comprensión de fenómenos complejos, como por ejemplo, fenómenos físicos, ya que tienen la oportunidad de interactuar y de aprehenderlos de una forma que está fuera de las posibilidades de una práctica real.

En este marco, el uso de software como herramienta didáctica constituye una alternativa para los docentes, pues facilita el trabajo de modelación de fenómenos físicos, lo cual se torna muy difícil usando tan sólo lápiz y papel. Esta herramienta posibilita expresar las fórmulas, las tablas, las gráficas y las simulaciones de forma rápida y confiable, optimizando el tiempo empleado y facilitando el análisis de los temas tratados, lo cual redunda en un sinnúmero de posibilidades para la labor docente. Por otra parte, el software como herramienta pedagógica incentiva a los estudiantes a hacer conjeturas, generar hipótesis, enunciar y calcular eventos a partir de una estrategia de enseñanza aprendizaje. Igualmente, facilita el trabajo para establecer patrones, variables, propiedades numéricas, geométricas y algebraicas en torno a un tema abordado. Todo ello se reitera en la cualificación de procesos de pensamiento analítico y algorítmico.

\section{Tipos de software}

Existen varias posibilidades en el uso de software como herramienta pedagógica; a continuación se presentan dos alternativas: el software propietario y el software libre.

\section{El software propietario}

Son aquellos programas que tienen restricciones para su uso, distribución, modificación o redistribución, pues tienen derechos de autor, razón por la cual su código fuente no está abierto o disponible y el derecho a uso está supeditado al pago de la licencia correspondiente. Los altos costos de las licencias y de sostenibilidad para cada equipo dificultan su uso masivo. En el caso de los centros educativos resulta un cargo oneroso. Algunas ventajas del software propietario ${ }^{2}$ son:

a. La licencia incluye soporte técnico para todo tipo de hardware.

b. Reduce la demanda de técnicos especializados.

c. La mayoría de las aplicaciones utilizadas en el mercado son software propietarios.

d. Se destina una parte importante de los recursos a la investigación sobre la usabilidad del producto.

\section{El software libre}

Generalmente, el software libre ${ }^{3}$ se asocia con gratuidad, es decir, sin costo. Pero esto es erróneo, pues su filosofía consiste en que todos los usuarios tengan la libertad de cumplir cuatro leyes fundamentales: ejecutarlo, copiarlo, estudiarlo y mejorarlo. En este caso, lo libre se refiere a la posibilidad de modificar el código fuente y no al costo de la licencia. El espíritu colaborativo de este tipo de software permite que cada persona lo utilice y lo desarrolle, adecuándolo según sus propias necesidades. Cada mejora realizada debe publicarse de manera que otros puedan, a su vez, utilizar las versiones resultantes de esta actividad y hacer nuevas mejoras, contribuyendo de esta forma a un mayor desarrollo de los programas de software, restando impulso a la piratería.

Algunas ventajas del uso del software libre en la educación son:

\footnotetext{
2. Culebro, M., Gómez, W. y Torres, S. (2006). Software libre vs software propietario: Ventajas y desventajas. Recuperado en julio de 2010, desde: http://gnutransfer.info/descargas/software_libre_vs_propietario.pdf.

3. Stallman, R. M. (2007). Software libre para una sociedad libre. Recuperado en julio de 2010, desde: http://www.gnu.org/philosophy/fsfs/ free_software.es.pdf.
} 
a. Se puede adaptar a los equipos ya existentes.

b. No exige el uso de equipos sofisticados.

c. No requiere de grandes presupuestos para su implementación.

d. Está constituido por programas livianos, es decir, no ocupa mucho espacio en disco duro.

e. Son programas robustos, ya que contienen una alta gama de aplicaciones.

f. Fácil acceso, se puede descargar de Internet de forma rápida y segura.

Por tal motivo, el software libre constituye una buena alternativa para los centros educativos, porque no se requieren equipos sofisticados ni grandes presupuestos; además, posibilitan el acceso a recursos que favorecen el desarrollo de las competencias académicas y laborales exigidas en el mundo actual. Podemos encontrar una amplia gama de software en la red. Entre las muchas posibilidades mencionamos las siguientes alternativas ${ }^{4}$ :

\begin{tabular}{|c|c|}
\hline Tipo & Programas \\
\hline & Axiom \\
& YACAS \\
Programas de cálculo & GiNaC \\
simbólico & Pari \\
& Maxima \\
& wxMaxima \\
& XMaxima \\
\hline & EULER \\
Programas de cálculo & Octave \\
numérico y matricial & R \\
& Scilab \\
& Tela \\
\hline Interfaces de usuario & TEXmacs \\
genéricas & WIMS \\
\hline
\end{tabular}

La alternativa de software escogida para desarroIlar en el manual fue Scilab, por su entorno amigable y su similitud con paquetes de Matlab y Maple. Aunque la razón primordial fue continuar con un proceso anterior, iniciado con la elaboración de un manual titulado Fundamentos de Scilab y aplicación, en la Licenciatura en Electrónica de la Universidad Pedagógica Nacional.

\section{Scilab}

Es un paquete con licencia GPL o licencia pública general, que usan la mayoría de los programas de GNU y más de la mitad de los paquetes de software libre. Scilab ${ }^{5}$ es una extensión del software propietario Matlab y tiene un lenguaje de programación de alto nivel, es decir, que permite realizar cualquier tipo de algoritmos o programación. Tiene la ventaja de que es liviano, robusto y permite realizar varios cálculos numéricos en aplicaciones académicas y científicas. Fue desarrollado por el grupo INRIA de Francia en 1992. Posee varias aplicaciones y herramientas, tales como matrices, operaciones numéricas, polinomios, sistemas lineales, tratamiento de señales, sistemas de control, editor gráfico en 2D y 3D, Scicos, animación y simulación.

Scicos ${ }^{6}$ es una extensión de Scilab, similar a la que posee Matlab, Ilamada Simulink $(2010)^{7}$, que permite realizar simulaciones y análisis de sistemas dinámicos. Utiliza bloques gráficos para evaluar y modificar sistemas. Scicos adquirió importancia debido a su amplia gama de aplicaciones preprogramadas; en él podemos hacer análisis empleando diagramas de bloques aplicados al modelamiento de diversos sistemas, especialmente los dinámicos, y adicionalmente permite compilar modelos en modo ejecutable.

Sus principales características son:

- Permite modelar, compilar, simular y graficar, a través de bloques, sistemas dinámicos en tiempo continuo y discreto.

- Los bloques son compatibles con lenguajes de programación como C, FORTRAN, o su propia sintaxis.

- Las simulaciones funcionan en tiempo real con los dispositivos verdaderos usando Scicos-HIL.

- Genera los ejecutables en tiempo real empleando la herramienta de Scicos/RTAI.

- Permite elaborar simulaciones en sistemas de comunicaciones digitales con Scicos-ModNum.

\footnotetext{
4. Bustos Pérez, J. Á., Peralta de Arriba, L. (2008). GNU/Linux, software libre para la comunidad universitaria software cientifico en GNU/ Linux. Recuperado en julio de 2010, desde: http://www.augcyl.org/doc/2008-curso-salamanca/07-software_cientifico.pdf.

5. Scilab is trademark of INRIA (1989). Recuperado en marzo de 2010, desde: http://www.scilab.org/

6. Recuperado en marzo de 2010, desde: http://www.worldlingo.com/ma/enwiki/es/Scicos

7. Leyva Rodríguez, C. Manual de simulación para sistemas de control usando el software Matlab y Scilab. Universidad Veracruzana, Facultad de Ingeniería Mecánica Eléctrica. Recuperado en mayo de 2010, desde: www.scilab.org/contrib/download.php
} 


\section{Pre.Impresos $\underset{\text { Bstudiantes }}{6}$}

\section{Desarrollo}

Este manual es un instrumento de apoyo al docente para diseñar talleres y actividades exploratorias de tipo descriptivo que ayuden a los estudiantes a afianzar los conceptos desarrollados en clase. Las actividades planteadas permiten manipular variables y valores, determinando lo que sucede con la simulación, de modo que quienes realicen las prácticas puedan generar hipótesis respecto a un fenómeno físico estudiado. Además se pueden realizar en la casa o en el aula.

Algunas ventajas de realizar estas prácticas por medio de una simulación son:

- Permite desarrollar un pensamiento algorítmico y lógico en la realización de los procesos matemáticos.

- Estimula la aproximación del estudiante al pensamiento científico e investigativo.

- Brinda un mejor apoyo para el desarrollo de las temáticas.

- Facilita herramientas poderosas para modelar la realidad.

- Da la posibilidad de hacer los cambios necesarios sin el riesgo de maltratar o dañar los instrumentos o equipos de laboratorio.

- Facilita al estudiante medir, calcular el error y sacar conclusiones.

- Posibilita anticiparse o enfrentarse a situaciones o eventos futuros.

- El estudiante puede elegir lo relevante para la realización de sus prácticas.

- Se reducen costos de montaje de las prácticas y de mantenimiento de elementos de los laboratorios.

- Se pueden hacer prácticas más estructuradas para el desarrollo de las competencias analíticas, propositivas y argumentativas.

La experiencia adquirida con el empleo de la simulación como parte de la práctica de laboratorio en los cursos de Comunicaciones I y II puso de manifiesto la importancia de Scilab como una alternativa para satisfacer requerimientos académicos, como el fortalecimiento del componente práctico de las clases y de optimización de recursos, por ejemplo, repetir las prácticas de laboratorio las veces necesarias para apoyar el aprendizaje de las temáticas vistas, sin incurrir en un incremento de los costos.
Por otro lado, al elaborar el manual con Scilab fuimos pioneros en desarrollar el primer texto en español para el uso de las herramientas de este software para un curso completo, resaltando los beneficios de las interfaces del editor, consola, sus componentes gráficos y de simulación para la realización de prácticas virtuales sobre los temas de Comunicaciones I y II. Aunque existían varios documentos referentes al funcionamiento y aplicación de Scilab, como por ejemplo el trabajo de grado Fundamentos de Scilab y Aplicación, desarrollado en el año 2000 en la Universidad Pedagógica Nacional de Colombia, hasta ese momento ninguno se había especializado en un tema que demandara el empleo de todas las herramientas del programa para su uso en una asignatura.

En un principio se contaba con una vasta información para la elaboración de gráficas y simulaciones con Scilab. De esta forma se comenzó a trabajar el proyecto de grado con la dirección del profesor Jimmy W. Ramírez Cano. En su asesoría se decidió tomar como guía el libro Elementos de electromagnetismos (Matthew N. Sadikus, 2003). Para la segunda parte se emplearon los libros Análisis de Fourier (Hwei P. Hsu, 2000), y Comunicaciones l: señales, modulación y transmisión (Enrique Herrera Pérez, 2004). Estos libros fueron utilizados como textos guías por los docentes en las asignaturas de Comunicaciones Iy II.

Los temas tratados en la asignatura de Comunicaciones I fueron los conceptos básicos de vectores y sistemas de coordenadas, que abordan la relación existente entre cada uno de ellos, que son esenciales para el trabajo en Cálculo Aplicado a Vectores y que permiten tener fundamentos de Electromagnetismo y de Matemáticas, necesarios paras las aplicaciones en los temas de Electroestática, Magnetostática y Ondas.

Para las temáticas de la asignatura de Comunicaciones II, se recogieron los conceptos básicos de Fourier en Transformada de Fourier para las aplicaciones en teoría de comunicaciones en señales moduladas. Se tienen dos tipos de modulación, la modulación análoga y la modulación digital. Para la señal análoga se trabajó AM (amplitud modulada), FM (frecuencia modulada) y los conceptos de señal modulada, portadora cuando es continua y espectros. Para la señal digital se trabajó FSK (modulación por corrimiento de frecuencia), PSK (modulación digital de fase), QAM (modulación por amplitud en 
cuadratura), ASK (modulación por corrimiento de amplitud), PCM (modulación por codificación de pulso) cuando la portadora es discreta.

Pese al propósito específico que lo animó, el manual puede ser utilizado para otras asignaturas que tengan los componentes de Física y Matemáticas que aquí se desarrollan y a la vez permite construir otras aplicaciones para temas diferentes que no se contemplaron en él. Otra parte importante de este documento es que en él se desarrolla toda una asignatura a partir de componentes gráficos y de simulación; esto permite que el estudiante pueda manipular las variables, modificar el código y experimentar sobre modelos físicos que se pueden presentar en la vida real y que no son tan fáciles de observar y analizar en una práctica real. Además, Scilab permitió desarrollar componentes temáticos de las asignaturas de Comunicaciones I y II, que hasta entonces no se incluían en las prácticas de apoyo en estas materias.
El documento elaborado tiene como título Manual de Scilab de apoyo en los cursos de Comunicaciones I y II para la Universidad Pedagógica Nacional. Está organizado de la siguiente forma: se trabajan los conceptos básicos del tema abordado para contextualizar al lector, ya sea docente o estudiante; se realiza un repaso de las bases matemáticas necesarias para el desarrollo de los temas; se construye el algoritmo en Scilab para generar la simulación y la gráfica a partir de los conceptos matemáticos, utilizando para ello un caso que sirva como ejemplo.

Por esto, el manual se constituye en un instrumento útil para los docentes y estudiantes, pues apoya los procesos de enseñanza y aprendizaje en los cursos de Sistemas de Comunicaciones I y II por medio de simulaciones.. 


\section{Pre.Impresos $\underset{\text { Bstudiantes }}{6}$}

\section{Manual}

El manual está estructurado de manera que propicie una mejor comprensión de los conceptos descritos y desarrollados. A continuación se presentan algunos ejemplos contenidos en el documento original y se describe cada uno de los dominios de representación tratados.

El primer tema es el concepto de vector, definido por los componentes de dirección, magnitud y ángulo. Su importancia radica en que a través de los vectores podemos modelar los fenómenos físicos como la fuerza, el desplazamiento, la velocidad y la aceleración, de gran utilidad para el estudio de los campos magnéticos y aplicaciones en sistemas de comunicaciones como redes, líneas de transmisión y antenas. Todo ello por medio de una representación gráfica en un sistema de coordenadas, ya sea en 2D o en 3D, por ejemplo: el vector resultante en $3 \mathrm{D}$ se grafica por medio de Scilab en las coordenadas cartesianas $(2,3,4)(4,5,2)$.

\section{Dominio gráfico}

\section{Paralelogramo}

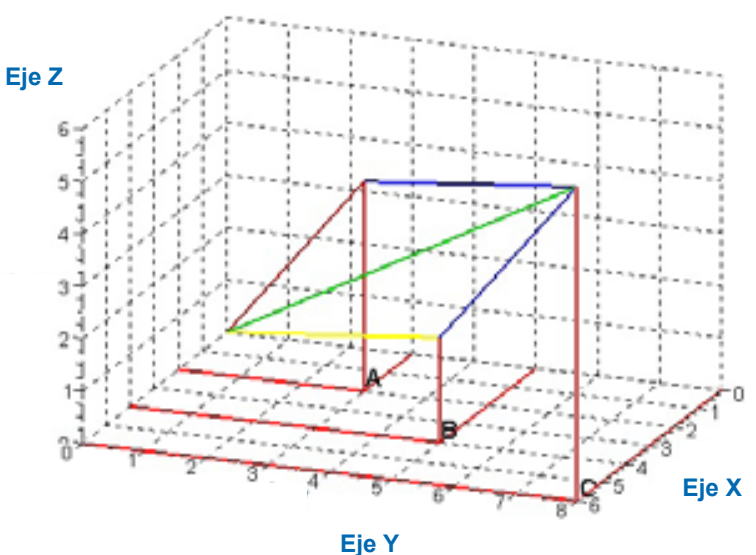

Figura 1

\section{Dominio analítico}

En este ejemplo podemos observar en la Figura 1 los componentes de los vectores en tres dimensiones, advertimos que el vector $A$ se encuentra representado por el color café y que éste a su vez tiene la misma magnitud del vector $\mathrm{BC}$ que está representado de color azul, además, que el vector $B$ se encuentra representado por el color amarillo, y que tiene la misma magnitud del vector AC. La suma del vector $A$ y $B$ genera un nuevo vector resultante $C$ en los puntos $(6,8,6)$. Podemos decir que el vector resultante es la diagonal de un paralelogramo que está conformado por los vectores A y B.

\section{Dominio simbólico}

La representación matemática de un espacio tridimensional lo simbolizamos como la matriz $3 \times 1$, la suma de los vectores $P(x 1, y 2, z 3)$ y de $Q(x 2, y 2, z 2)$ se representa en forma matricial como:

$$
a+b=\left|\begin{array}{l}
x 1+x 2 \\
y 1+y 2 \\
z 1+z 2
\end{array}\right|
$$

\section{Dominio instrumental}

A continuación se describe la parte algorítmica de sta matriz $3 \times 1$, la cual nos permitirá construir la |ráfica de los vectores y del paralelogramo a partir Il vector resultante.

\section{I/Nectores $(2,3,4)(4,5,2)$}

$a=\left[\begin{array}{ll}2 & 3\end{array}\right]$ ]// vector $A$ se puede manipular los valores

$b=\left[\begin{array}{l}4 \\ 5\end{array}\right.$ 2];//vector $B$ se puede manipular los valores

$c=a+b ; / / v e c t o r$ resultante $c$

$\mathrm{x}=[0 \mathrm{a}(1)] \mathrm{y}=[0 \mathrm{a}(2)] \mathrm{z}=[0 \mathrm{a}(3)] \mathrm{j} / /$ componente del vector $\mathrm{A}$

$\mathbf{x} 1=[0 \mathrm{~b}(1)] ; y \mathbf{1}=[0 \mathrm{~b}(2)] ; z 1=[0 \mathrm{~b}(3)] ; / /$ componente del vector $B$

$\mathrm{x} 2=[0 \mathrm{c}(1)] ; \mathrm{y} 2=[0 \mathrm{c}(2)] ; z 2=[0 \mathrm{c}(3)]] / / /$ componentes del vector resultante $\mathrm{C}$

$\mathrm{x} 3=[\mathrm{a}(1) \mathrm{c}(1)] \mathrm{i} \mathbf{y} 3=[\mathrm{a}(2) \mathrm{c}(2)] \mathrm{z} 3=[\mathrm{a}(3) \mathrm{c}(3)] ; / /$ unión de los puntos $\mathrm{A} \mathrm{y} \mathrm{C}$

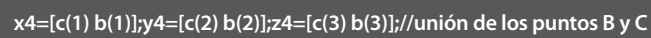

$\mathrm{x} 5=[\mathrm{a}(1) \mathrm{a}(1)] \mathrm{j} 5 \mathrm{5}=[0 \mathrm{a}(2)] \mathrm{z} 5=[00 \mathrm{0}] / / / \mathrm{Eje} \mathrm{y}$ del vector $\mathrm{A}$

$\mathrm{x} 6=[\mathrm{a}(1) \mathrm{a}(1)] \mathrm{y} \mathbf{6}=[\mathrm{a}(2) \mathrm{a}(2)] \mathrm{z} \mathbf{z}=[0 \mathrm{a}(3)] \mathrm{j} / / \mathrm{Eje} \mathrm{z}$ del vector $\mathrm{A}$

$\mathrm{x} 7=[\mathrm{a}(1)$ 0];y7 $=[\mathrm{a}(2) \mathrm{a}(2)] \mathrm{z} 7=[0$ 0];//Eje $\mathrm{x}$ del vector $\mathrm{A}$

$x 8=[b(1) b(1)] ; y 8=[0 b(2)] ; z 8=[000] / / /$ Eje $y$ del vector $B$

$x 9=[0 b(1)] ; y 9=[b(2) b(2)] ; z 9=[000] ; / / / j e x$ del vector $B$ 
$\mathrm{x} 10=[\mathrm{b}(1) \mathrm{b}(1)] \mathrm{y} 10=[\mathrm{b}(2) \mathrm{b}(2)] ; z 10=[\mathrm{b}(3)$ 0];//eje $z$ del vector $\mathrm{B}$

$\mathrm{x} 11=[\mathrm{c}(1) \mathrm{c}(1)] ; \mathrm{y} 11=[0 \mathrm{c}(2)] ; z 11=[0$ 0];// Eje $\mathrm{y}$ del vector $\mathrm{C}$

$\mathrm{x} 12=[0 \mathrm{c}(1)] \mathrm{y} \mathbf{1 2}=[\mathrm{c}(2) \mathrm{c}(2)] \mathrm{z} 12=[0$ 0]///Eje $\mathrm{x}$ del vector $\mathrm{C}$

$x 13=[c(1) c(1)] ; y 13=[c(2) c(2)] ; z 13=[0 c(3)] ; / / E j e z$ del vector $C$

//Comandos que genera un gráfica 3D

plot3d3 $[$ [x], [y], [z])//vector A

plot3d3([x1],[y1],[z1])// vector B

plot3d3([x2],[y2],[z2])//vector resultante C

plot3d3([x3,x4],[y3,y4],[z3,z4])//uniòn de los vector

plot3d3([x5,x6,x7],[y5,y6,y7],[z5, z6,z7])///ejes del vector $A$

plot3d3([x8,x9,x10],[y8,y9,y10],[z9,z8,z10])// ejes del vector B

plot3d3([x11,x12,x13],[y11,y12,y13],[z11,z12,z13])///ejes del vector $C$

plot3d1 $(x, y, z$, leg="eje x@ejey@eje z")

xgrid(1); $\operatorname{xgrid(3);~}$

xstring(a(1),a(2),",A");

xstring(b(1),b(2),"B"

xstring(c(1),c(2),"A X B")

\section{El dominio lingüístico}

Este dominio se emplea a lo largo de los demás dominios, ya que son concurrentes las expresiones de vector, vector resultante, paralelogramo, magnitud, dirección, vector 3D, componentes del eje $x, y, z$ y sistemas coordenadas.

La primera fase del manual desarrolla temáticas del sistema de comunicación I, empleando los dominios de representación de forma similar a los ejemplos anteriormente mencionados. Cabe aclarar que los dominios se pueden trabajar de forma aleatoria e independiente, lo que permite al docente desarrollar actividades que fortalezcan las habilidades del estudiante según el dominio que se quiera trabajar o desarrollar a través de su exploración. De igual forma, el estudiante adquiere las habilidades que demanda su trabajo a través de los análisis y las conclusiones; esto permite que adquieran un aprendizaje significativo, logrando su propio conocimiento $y$, a su vez, evidenciando su aprendizaje.

Un ejemplo corresponde al tema de Cálculo Aplicado a Vectores. Su importancia radica en la aplicación de las diferenciales en sistemas de comunicaciones, por ejemplo, en la descripción de las trayectorias que se producen sobre una línea, superficie o volumen, en un campo eléctrico producido por una carga lineal, para lo cual se desarrollan los teoremas de Gauss y de Stokes, permitiendo describir la carga total que existe en un cuerpo o en un flujo de corriente.

El ejercicio se ilustra en la Figura 2, en donde se muestra un plano de coordenadas esféricas que tienen los puntos $D=\left(5, \frac{\pi}{2}, 2 \pi\right)$. Encontrar el volumen diferencial a lo largo de $r, \theta$ y $\varnothing$.

\section{Dominio gráfico}

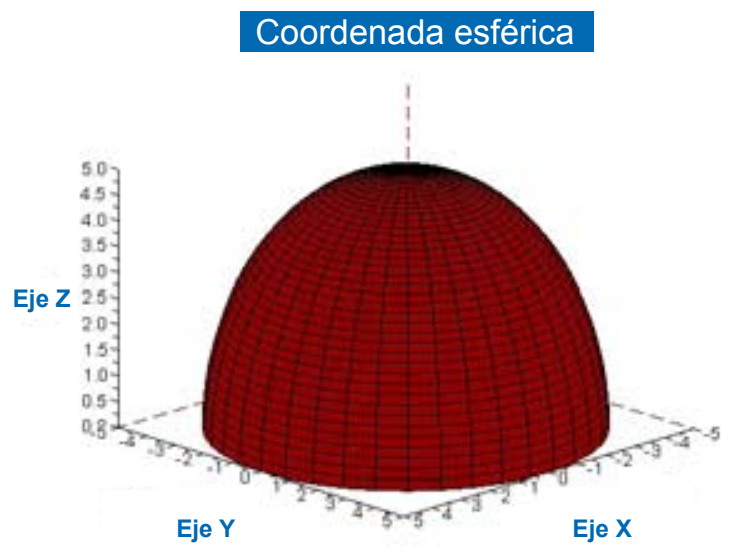

Figura 2

\section{Dominio analítico}

Observamos la Figura 2, una semiesfera que está construida por las coordenadas $r$, theta y phi, donde $r$ es el radio de la esfera, el ángulo theta y el ángulo de phi son los desplazamientos angulares. El ángulo theta tiene el valor de $\frac{\pi}{2}$ radianes en las coordenadas $z$ y $x$, el ángulo phi con el valor de $2 \pi$ radianes en las coordenadas $x$ y $y$.

El volumen diferencial ${ }^{8}$ se halla resolviendo la integral de:

$$
\int_{0}^{5} \int_{0}^{\frac{\pi}{2}} \int_{0}^{2 \pi} r^{2} \sin (\theta) d r d \theta d \emptyset
$$

\footnotetext{
${ }^{8 .}$ La función seno se define como se haría en un algoritmo en Scilab, de la forma $\sin (\Theta)$.
} 


\section{Pre.Impresos $\underset{\text { Bstudiantes }}{6}$}

Con el algoritmo podemos apreciar que la primera integral corresponde a términos de dr en los límites de 5 a 0 , la segunda integral corresponde a términos de $d \varnothing$ en los límites de $\frac{\pi}{2}$ a 0 y la tercera integral corresponde a términos de $\mathrm{d} \theta$ en los límites de $2 \pi$ a 0 . Las integrales se realizan por separado y los resultados se multiplican para obtener el valor del volumen de la semiesfera:

$$
v=261.79939
$$

\section{Dominio simbólico}

El volumen diferencial se expresa de la siguiente manera:

$$
d v=r^{2} \sin (\theta) d r d \theta d \emptyset
$$

\section{Dominio instrumental}

A continuación se describirá el algoritmo que nos permite elaborar la semiesfera.

xset("colormap",hotcolormap(21))

//Esfera.

theta $=$ linspace $(0, \%$ pi $/ 2,40) ; / /$ ángulo theta en radianes

phi $=$ linspace $(0,2 * \%$ pi,40);//ángulo phi en radianes

$r=5 ; / /$ radio de la esfera

$x=r^{*}\left(\sin (\text { theta })^{* *} \cos (\right.$ phi $\left.)\right)$;

$y=r^{*}\left(\sin (\text { theta })^{\prime *} \sin (\right.$ phi $\left.)\right)$;

$z=r^{*} \cos (\text { theta })^{\prime *}$ ones(theta);//ones es una matriz de unos

$[X, Y, Z]=n f 3 d(x, y, z) ; / / n f 3 d$ permite un transformación

//rectangular en las tres coordenadas de la matriz

plot3d1 (X,Y,Z,leg="eje x@eje y@eje z")

Este algoritmo permite hallar el valor del volumen de la semiesfera:

Nolumen diferencial esférico

dr=integrate('( $\left.(r \wedge 2), r^{\prime}, 0,5\right)$;

dtheta=integrate('(sin(theta)),"theta',0,\%pi/2);

dphi=integrate('(1),"phi',0,2*\%pi);

$\mathrm{v}=\mathrm{dr}^{*} \mathrm{dtheta}{ }^{*} \mathrm{dphi}$

\section{Dominio lingüístico}

En este dominio prevalece a lo largo del texto. Se manifiesta en el uso adecuado de términos como coordenadas esféricas, integración, volumen, ángulo theta y phi.

La profundización del tema permite retomar elementos conceptuales como área, volumen y desplazamiento en diferencia, donde se desarrollan integrales y derivadas. Para la realización de las integrales se empleó la instrucción: integrate ('funcion'; 'variable a evaluar'; $\lim \times 0$; $\lim \times 1$ ), donde se introduce la función que se va a integrar, luego la variable por la cual se desea integrar y los límites de integración. 


\section{Sistemas de Comunicaciones II}

En la segunda fase, el manual continúa con la misma estructura que se trabajó en la primera, a través de los dominios de representación que se emplearon anteriormente en el documento. La temática más relevante tratada en Sistemas de Comunicaciones II es la modulación; un caso particular es la modulación en amplitud o AM que trabaja las señales continuas de la moduladora, la portadora, la modulada y su espectro.

Por ejemplo, se tiene una señal AM que contiene una frecuencia de $2,5 \mathrm{Khz}$ con una amplitud pico de $2 \mathrm{~V}$ y se modula con una señal seno con una frecuencia de $250 \mathrm{Khz}$ con un pico de $1 \mathrm{~V}$. Con el ejercicio se propone que el estudiante determine el espectro de la señal e identifique la ubicación de las señales portadora, moduladora y modulada.

\section{Dominio gráfico}

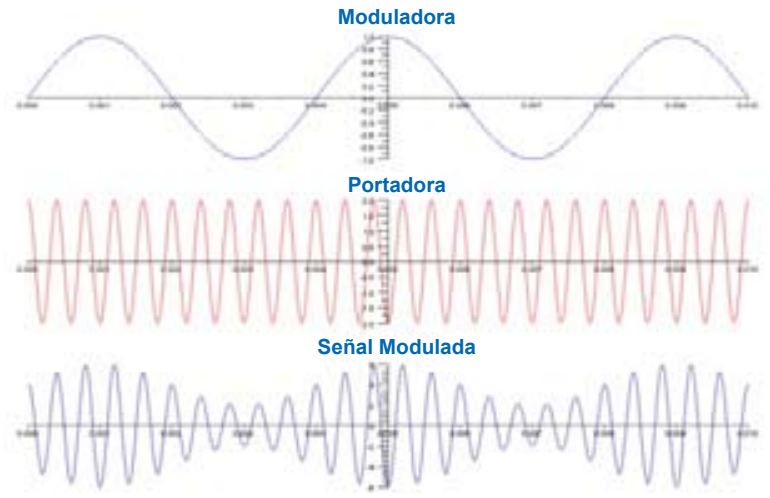

Figura 3

\section{Dominio analítico}

En la Figura 3 podemos observar la señal moduladora, portadora y la modulada. La señal moduladora es donde se encuentra la información o el mensaje, la señal portadora debe ser de alta frecuencia para que permita llevar la información y la señal modulada corresponde a la convolución, es decir, la multiplicación de moduladora y portadora.

\section{Dominio simbólico}

En esta gráfica observamos que en la primera figura la señal corresponde a la moduladora, que se expresa de la siguiente manera:

$$
m(t)=1^{\star} \sin \left(w_{m} t\right)
$$

En la segunda figura se observa la señal portadora, que se expresa de la siguiente manera:

$$
c(t)=A c^{\star} \cos \left(w_{c} t\right)
$$

Y por último, la señal moduladora, que se representa así:

$$
f(t)=A c[1+m(t)] \cos \left(w_{c} t\right)
$$

Por consiguiente, la expresión de la señal moduladora es:

$f(t)=2\left[1+0.5^{\star} \sin (500 \pi k h z) t\right] \cos ((5 \pi k h z) t)$

\section{Dominio instrumental}

El valor de $m=0,5$, que corresponde al índice de modulación donde $m<1$. Además, debemos tener claro que la frecuencia de la portadora es mayor que la moduladora, es decir, wc $>$ wm.

A través de Scilab podemos elaborar la señal modulada, la señal portadora y la señal moduladora:

\section{//Modulación AM}

$\mathrm{n}=2000$; // número de divisiones

ini $=0$; // tiempo de inicio

fin $=0.015$; //tiempo final

inte=(fin-ini)/n; // tiempo de intervalos

$\mathrm{t}=[$ ini:inte:fin]; // tiempo para generar las gráficas

$\mathrm{fc}=2500$;//frecuencia portadora

$\mathrm{fm}=250 ; / /$ frecuencia moduladora

Ac=2;//valor pico de portadora

$\mathrm{K}=1 ; / /$ valor pico de la moduladora

$\mathrm{m}=\mathrm{K} / \mathrm{Ac} / /$ indice de modulación

$\mathrm{mt}=K^{*} \sin \left(2^{*} \% \mathrm{pi}^{*} \mathrm{fm}{ }^{*} \mathrm{t}\right) ; / /$ moduladora 


\section{Pre.Impresos $\underset{\text { Estudiantes }}{6}$}

subplot(311);plot2d(t,mt,[2]) // imagen tres en una sola pantalla en la

primera ubicación

$\mathrm{a}=\mathrm{gca}()$;

a.x_location="middle";

a.y_location="middle";

xtitle("MODULADORA")

$\mathrm{ct}=\mathrm{Ac} c^{*} \cos \left(2^{*} \% \mathrm{pi}^{*} \mathrm{fc} * \mathrm{t}\right) ; / /$ portadora

subplot(312);plot2d(t,ct,[5]) // Imagen tres en una sola pantalla en la

segunda ubicación

$\mathrm{a}=\mathrm{gca}()$;

a.x_location="middle";

a.y_location="middle";

xtitle("PORTADORA")

$\mathrm{ct}=\mathrm{Ac}{ }^{*} \cos \left(2 * \% \mathrm{pi}^{*} \mathrm{fc} * \mathrm{t}\right) ; / /$ portadora

sam $=A c^{*}(1+m . * m t) . * t ; / /$ señal AM

subplot(313);plot2d2(t,sam,[9]) // tres imágenes en una sola pantalla en la

tercera ubicación

$\mathrm{a}=\mathrm{gca}()$;

a.x_location="middle";

a.y_location="middle";

xtitle("SEÑAL MODULADA")

\section{Dominio lingüístico}

En este dominio, los términos que se manejan a lo largo de este capítulo son señal moduladora, señal portadora y señal modulada.

Para continuar con el desarrollo del ejercicio se presenta la gráfica correspondiente al espectro de AM:

\section{Dominio gráfico}

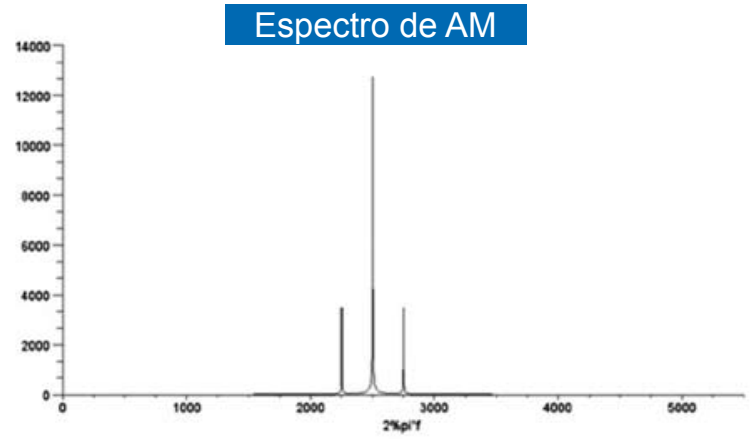

Figura 4

\section{Dominio analítico}

Podemos observar en la Figura 4 como el espectro de una señal modulada en amplitud tiene un pulso que se encuentra localizado en la frecuencia $W_{c} y$ otros pulsos que son el espectro de $\mathrm{m}(\mathrm{t})$ alrededor de $W_{c}$ y se denomina bandas laterales superior e inferior a las llevan la información de la señal y las podemos observar en la Figura 4. Recordemos que la frecuencia $W_{c}$ de la señal AM que contiene una frecuencia de $2,5 \mathrm{Khz}$ y que la frecuencia de $\mathrm{m}(\mathrm{t})$ se modula con una señal seno con una frecuencia de $250 \mathrm{Khz}$.

\section{Dominio simbólico}

Para encontrar el espectro de la señal modulada utilizamos el teorema de translación de la frecuencia, el cual dice que al multiplicar una señal $f(t)$ por una señal sinusoidal de frecuencia $W_{c}$ traslada su espectro en $\pm W_{c}$; tenemos la siguiente expresión:

$$
\begin{gathered}
F(w)=F[f(t)] \\
F(w)=F\left[A_{c} * \cos \left(\left(w_{c}\right) t\right)\right]+F\left[A_{c} * m(t) \cos \left(\left(w_{c}\right) t\right)\right]
\end{gathered}
$$

$F(w)=A_{c} \pi \delta\left(w-w_{c}\right)+A_{c} \pi \delta\left(w+w_{c}\right)+\frac{1}{2} A_{c} M\left(w-w_{c}\right)+\frac{1}{2} A_{c} M\left(w+w_{c}\right)$

\section{Dominio instrumental}

Para determinar $F[m(t)]=M(w)$ se emplea la siguiente instrucción en Scilab:

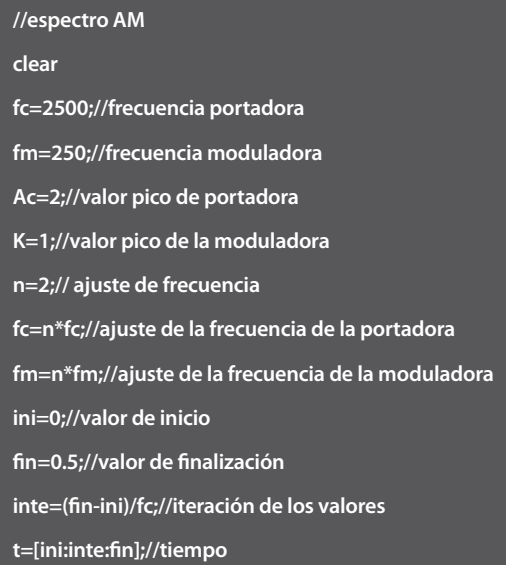


$\mathrm{m}=\mathrm{K} / \mathrm{Ac} ; / /$ índice de modulación

$\mathrm{mt}=K^{*} \sin \left(2 * \% \mathrm{p}^{*} \mathrm{fm}^{*} \mathrm{t}\right)$ )//moduladora

$\mathrm{ct}=\mathrm{Ac} * \cos \left(2^{*} \% \mathrm{pi}^{*} \mathrm{fc} * \mathrm{t}\right) ; / /$ portadora

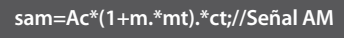

$\mathrm{h}=\mathrm{fft}(\mathrm{sam}) ; / /$ transformada rápida de Fourier

plot2d2(abs(h))

xtitle("Espectro de AM"','2\%pi*f")

\section{Dominio lingüístico}

En este dominio, los términos que se manejan a lo largo de este capítulo de Sistemas de Comunicaciones Il son: señal moduladora, señal portadora y señal modulada, espectro, banda inferior, banda superior.

En el documento final se desarrollan temas como señal de AM con portadora suprimida y señal de AM con banda lateral única. De igual forma, se trabajó la frecuencia modulada FM, donde se observó el comportamiento y la gráfica de la señal de la portadora, la señal de la moduladora y la señal FM modulada.

El último tema tratado en este manual fue modulación digital, ASK (modulación por corrimiento en amplitud), FSK (modulación por corrimiento de frecuencia), PSK (modulación digital de fase), QAM (modulación por amplitud en cuadratura), PCM (modulación por codificación de pulso), temas que son trabajados de la misma forma que los ejemplos anteriores.

\section{Conclusiones}

- El empleo de software como herramienta de apoyo didáctico ha permitido mejorar las prácticas de laboratorio para desarrollar aquellas competencias profesionales requeridas en el mundo académico y laboral.

- El uso del manual como apoyo a los docentes de la asignatura de sistemas de comunicación ha permitido desarrollar prácticas de laboratorio, abordando a cabalidad los componentes temáticos de las asignaturas.

- El uso de Scilab como herramienta didáctica facilita la elaboración de laboratorios virtuales, cuando no haya condiciones para realizar prácticas reales en el aula.

- El desarrollo de los ejercicios a través de los dominios de representación es coherente con las instrucciones empleadas a través de Scilab para integrarlos y presentarlos en forma de simulación y gráfica, de manera que el estudiante debe emplear e interpretar los algoritmos desarrollados para hacer posteriores modificaciones o construcciones, estimulando el pensamiento algorítmico.

- El uso adecuado de las TIC facilita los procesos de enseñanza y puede aportar nuevas herramientas para la producción de nuevas investigaciones, fomentar el trabajo cooperativo, generar un aprendizaje significativo dejando de lado lo memorístico. Además, las actividades son más interactivas.

- Se abren nuevos escenarios de aprendizaje, dando mayor autonomía e independencia al estudiante en su propio proceso de conocimiento.

Se puede encontrar información complementaria y el manual completo en la dirección URL http://ohra.zobyhost.com/. 


\section{Pre.Impresos $\underset{\text { Bstudiantes }}{6}$}

\section{Acerca de Scilab}

Scilab es un software matemático, con un lenguaje de programación de alto nivel, para cálculo científico, interactivo, de libre uso y disponible en múltiples sistemas operativos (Mac OS X, GNU/Linux, Windows), desarrollado en 1990 por investigadores del INRIA (Institut National de Recherche en Informatique et Automatique) y de la ENPC (École Nationale des Ponts et Chaussées). El Consorcio Scilab (Scilab Consortium en inglés) fue creado en mayo de 2003 para ampliar y promover Scilab como software de referencia, particularmente en el mundo académico y la industria. En julio de 2008, con el fin de mejorar la transferencia de tecnología, el Consorcio Scilab se unió a la Fundación Digiteo.

Scilab fue creado para hacer cálculos numéricos, aunque también ofrece la posibilidad de hacer algunos cálculos simbólicos, como derivadas de funciones polinomiales y racionales. Posee cientos de funciones matemáticas y la posibilidad de integrar programas en los lenguajes más usados (Fortran, Java, C y C++). Scilab fue hecho para ser un sistema abierto donde el usuario puede definir nuevos tipos de datos y operaciones entre los mismos.

Viene con numerosas herramientas, como gráficos 2D y 3D, animación, algebra lineal, matrices dispersas, polinomios y funciones racionales; simulación: programas de resolución de sistemas de ecuaciones diferenciales (explícitas e implícitas); Xcos: simulador por diagramas en bloque de sistemas dinámicos híbridos; control clásico, robusto, optimización LMI; optimización diferenciable y no diferenciable; tratamiento de señales; grafos y redes; Scilab paralelo empleando PVM; estadísticas; creación de GUls; interfaz con el cálculo simbólico (Maple, MuPAD) e interfaz con TCL/TK.

Además se pueden agregar numerosas herramientas hechas por los usuarios, como Grocer, una herramienta para Econometría u Open FEM (Una caja de Herramientas para Elementos Finitos), desarrollada por INRIA.

En el pasado, Scilab podía ser utilizado en el análisis de sistemas, pero no podía interactuar con el exterior. Hoy en día se pueden construir interfaces para que desde Scilab se pueda manejar un dispositivo, se conecte a la red a través de TCP (Protocolo de Control de Transmisión) o UDP (User Datagram Protocol), etc. Esto brinda la posibilidad de conectar una placa de adquisición de datos a Scilab y de esta forma el control de una planta on-line.

\section{Scicos}

La primera versión de Scicos fue escrita enteramente en lenguaje Scilab por R. Nikoukhah y no tenía editor gráfico. Más tarde, desarrolló el simulador de código en FORTRAN y algunas partes del compilador en C para el primer lanzamiento oficial en 1994, que incluía un editor gráfico realizado principalmente por S. Steer.

Scicos fue desarrollado con la ayuda de estudiantes de doctorado, investigadores posdoctorales y pasantes hasta finales de los 90 . El generador de código fue desarrollado, por ejemplo, por Rachid Djenidi, estudiante de doctorado, y una implementación optimizada del compilador fue realizado por Azzedine Azil, también estudiante de doctorado. Los socios industriales proporcionaron ejemplos de aplicación e incluso participaron activamente en los desarrollos.

\section{ScicosLab}

Cuando el Consorcio Scilab lanzó el desarrollo de Java basada en Scilab 5 en el año 2005, el equipo del proyecto Metalau y el ENPC decidieron mantener su propia versión de Scilab, basada en Scilab 4, para la distribución de los nuevos desarrollos de software procedente de las actividades de investigación del equipo Metalau en INRIA y ENPC.

Aunque llamada inicialmente ScilabGtk, esta versión se denominó más tarde ScicosLab, para evitar cualquier confusión con el software Scilab. ScicosLab contiene las últimas versiones de MaxPlus y Scicos, que son cajas de herramientas desarrolladas en el proyecto Metalau.

Información tomada de la página de Sicos de INRIA. Recuperado en julio del 2010, de

http://www-scicos.inria.fr/ScicosModNum/modnum_web/ web/eng/eng.htm 


\title{
Sobre el Autor
}

\author{
Manuel Francisco Romero, Licenciado en Elec- \\ trónica de la Universidad Pedagógica Nacio- \\ nal de Colombia, graduado en el año 2008. \\ Realizó el curso de formación en Tutoría en \\ Ambiente Virtual de Aprendizaje ofrecido \\ por la Universidad Autónoma de Bucaraman- \\ ga, UNAB Virtual, y Universidad Tecnológica \\ de Bolívar. Ha trabajado en el desarrollo de \\ aplicaciones basadas en software libre como \\ apoyo académico y en la implementación de \\ asignaturas virtuales a través de la platafor- \\ ma Moodle, en la Fundación de Educación \\ Superior INSUTEC. Pertenece al grupo de \\ Investigación RIEMANN, que investiga sobre \\ las estrategias de enseñanza aprendizaje em- \\ pleando las Tecnologías de la Información y la \\ Comunicación (TIC). Participó en el Congreso \\ Iberoamericano de Educación realizado en \\ Argentina en Septiembre de 2010. Fue invi- \\ tado a mostrar su trabajo de investigación \\ titulado Las Tecnologías de la Información \\ y la Comunicación (TIC) como estrategia de \\ enseñanza-aprendizaje en la educación por \\ ciclos propedéuticos a la Universidad Católi- \\ ca de Chile, Universidad Santiago de Chile y la \\ Universidad Alberto Hurtado de Chile.
}

\section{w w w.p e d a gogica.e d u.co}

